\title{
Bcl-2 cleavages at two adjacent sites by different caspases promote cisplatin-induced apoptosis
}

\author{
Jianbei Zhu ${ }^{1}$, Ying Yang ${ }^{1}$, Jiarui $\mathrm{Wu}^{1,2}$ \\ ${ }^{1}$ Key Laboratory of Systems Biology, Institute of Biochemistry and Cell Biology, Shanghai Institutes for Biological Sciences, Chinese \\ Academy of Sciences, 320 Yueyang Road, Shanghai 200031, China; ${ }^{2}$ Hefei National Laboratory for Physical Sciences at Microscale \\ and School of Life Sciences, University of Science \& Technology of China, Hefei 230027, China
}

The protein encoded by $b c l-2$ proto-oncogene plays an important role in the mitochondria-mediated apoptotic pathway. Although the general role of Bcl-2 is anti-apoptotic, previous work showed that Bcl-2 fragments cleaved by caspases could promote apoptotic process. We report herein that Bcl-2 protein was cleaved to produce two fragments of around $23 \mathrm{kDa}$ in human hepatocarcinoma BEL-7404 cells or in Bcl-2 overexpressing CHO cells induced by cisplatin. Treating cells with the general caspase inhibitor Z-VAD-fmk blocked the induced cleavage of Bcl-2. Mutagenesis analyses showed that Bcl-2 was cleaved by caspases at two adjacent recognition sites in the loop domain (YEWD $\left.{ }^{31} \downarrow \mathrm{AGD}^{34} \downarrow \mathrm{V}\right)$, which could be inhibited by caspase- 8 and -3 inhibitors, respectively. Overexpression of the carboxyl terminal $23 \mathrm{kDa}$ fragments increased the sensitivity of $\mathrm{CHO}$ cells to cisplatin-induced apoptosis. These results indicate that Bcl-2 can be cleaved into two close fragments by different caspases during cisplatin-induced apoptosis, both of which contribute to the acceleration of apoptotic process.

Keywords: Bcl-2, apoptosis, cisplatin, caspase-3, caspase- 8

Cell Research (2007) 17:441-448. doi: 10.1038/cr.2007.36; published online 24 April 2007

\section{Introduction}

Apoptosis is a strictly controlled cellular process and plays an important role in the normal development of multicellular organisms as well as in pathological processes [1, 2]. There are two main pathways leading to apoptotic cell death. The extrinsic apoptotic pathway is activated by the interaction of death receptors on cell surface with their ligands, while the second pathway involves the participation of mitochondria and is regulated by members of the Bcl-2 family [3]. Anti-apoptotic members of Bcl-2 family such as Bcl-2 and Bcl-xL inhibit the release of cytochrome $\mathrm{c}$ from mitochondria, while pro-apoptotic members such as Bax and Bak decrease the mitochondrial membrane potential

\footnotetext{
Correspondence: Jiarui $\mathrm{Wu}$

Tel: +86 021 54921128; Fax: +8602154921011

E-mail: wujr@sibs.ac.cn

Received 19 November 2006; revised 19 January 2007; accepted 11 February

2007; published online 24 April 2007
}

$(\Delta \psi)$ and accelerate cytochrome $\mathrm{c}$ release to trigger downstream apoptotic events $[4,5]$.

The aspartate specific cysteine proteases called caspases amplify apoptosis by an activation cascade [6]. The activated caspases in turn cleave various cellular substrates, resulting in the characteristic biochemical and morphological changes associated with apoptosis [7]. For example, active caspase- 8 could cleave a pro-apoptotic Bcl-2 family member Bid to generate $\mathrm{tBid}$, which then translocates to mitochondria to facilitate cytochrome $\mathrm{c}$ release [8]. When caspase- 3 is activated, it cleaves several important proteins related to apoptosis such as the DNA repair enzyme poly(ADP-ribose) polymerase (PARP) [9] and DNA fragmentation factor 45 (DFF45) [10]. An increasing number of caspase substrates have been identified.

It has been reported that Bcl-2 is cleaved in the loop domain by caspase during apoptosis, which results in the inactivation of Bcl-2 [11] or even converts Bcl-2 to a Bax-like fragment [12]. Structural and functional analysis of the cleaved fragment revealed that the $\mathrm{NH}_{2}$-terminal 
region of Bcl-2 is required for its anti-apoptotic activity and heterodimerization with the pro-apoptotic protein Bax [13]. Inhibition of caspase-mediated cleavage of Bcl-2 results in suppression of apoptosis [14]. Some studies demonstrated that caspase- 3 was responsible for the Bcl-2 cleavage [11, 12 and 15]. However, Bcl-2 cleavage has been shown to occur in caspase-3 deficient MCF-7 cells [14]. Recently, our laboratory reported a caspase-8-dependent cleavage of $\mathrm{Bcl}-2$ in Chinese hamster ovary (CHO) cells [16].

In the course of examining cisplatin-induced apoptosis, we found that Bcl-2 was cleaved by different caspases at two adjacent recognition sites of $\mathrm{Asp}^{31}$ and $\mathrm{Asp}^{34}$ in the loop domain. Furthermore, both forms of the truncated Bcl-2 fragments increased the sensitivity of transfected cells to apoptotic stimulus.

\section{Materials and Methods}

\section{Cell culture}

CHO AA8 cells and human breast adenocarcinoma MCF-7 cells were grown in Dulbecco's modified Eagle's medium (DMEM, Gibco BRL) supplemented with $10 \%$ fetal bovine serum and $100 \mu \mathrm{M}$ nonessential amino acids (Gibco BRL). Stable subclone cells of CHO AA8 that overexpress dominant-negative caspase-8 (16) were maintained in complete medium containing $500 \mu \mathrm{g} / \mathrm{ml} \mathrm{G} 418$. Human hepatocarcinoma BEL-7404 cells were grown in DMEM medium supplemented with $10 \%$ fetal bovine serum.

\section{Plasmid construction}

The pEGFP vector containing wild-type human $b c l-2$ was described previous [16]. Substitutions of aspartic acids at 31 and 34 with alanine (D31A, D34A and D31, 34A) were accomplished by converting the aspartic acid codon GAT to alanine codon GCT by PCR mutagenesis. The following mutagenesis primers were used to generate $b c l-2$ site-directed mutants: 5'-TAC GAG TGG GA(C)T GCG GGA GA(C)T GTG GGC GCC-3' and 5'-GCC CCT CTG CGA CAG CTT ATA-3'. The $\mathrm{NH}_{2}$ terminal-truncated form of human Bcl-2 ( $\triangle \mathrm{N} 31$, lacking $\mathrm{NH}_{2}$-terminal 1-31 amino acids; $\Delta \mathrm{N} 31 \mathrm{D} 34 \mathrm{~A}, \Delta \mathrm{N} 31$ with a D34A mutation; and $\Delta \mathrm{N} 34$, lacking $\mathrm{NH}_{2}$-terminal 1-34 amino acids) were generated from pEGFP- $b c l-2$ by PCR and cloned into pcDNA4/TO vector (Invitrogen). The caspase-3 construct was generated by inserting human caspase- 3 cDNA into a pcDNA3.1 vector.

\section{Transient transfection}

$\mathrm{CHO}$ cells were transiently transfected with constructs expressing either the truncated Bcl-2 or full length human Bcl-2 fused to EGFP by electroporation with Nucleofector $\mathrm{T}$ kit according to the manufacturer's instructions (Amaxa Biosystems). Transfection of caspase-3 expression vector into $\mathrm{MCF}-7$ cells was conducted with lipofectamine 2000 (Invitrogen) according to the manufacturer's specifications. The transfection efficiencies were up to $70 \%$.

Preparation of cytosolic and mitochondrial extracts by digitonin treatment

CHO cells were harvested and resuspended in a buffer $(20 \mathrm{mM}$ Hepes-KOH, pH 7.3, $110 \mathrm{mM} \mathrm{KAc}, 5 \mathrm{mM} \mathrm{NaAc}, 2 \mathrm{mM} \mathrm{MgAc}_{2}$, and $1 \mathrm{mM}$ EGTA) containing $200 \mu \mathrm{g} / \mathrm{ml}$ digitonin (Calbiochem) on ice for $10 \mathrm{~min}$. The permeabilized cells containing cellular organelles and nuclei were pelleted as mitochondria-containing fractions, and the supernatants were collected as cytosolic fractions.

\section{Western-blotting analysis}

Intact cells, the supernatants, and the mitochondria-containing fractions were lysed in loading buffer $(50 \mathrm{mM}$ Tris- $\mathrm{HCl}, \mathrm{pH} 6.8$, $100 \mathrm{mM}$ dithiothreitol, $2 \%$ SDS, $10 \%$ glycerol, and $0.1 \%$ bromphenol blue). Equalized amounts of proteins from each sample were subjected to SDS-polyacrylamide gel electrophoresis (SDS-PAGE) and transferred to PVDF membrane. Western-blotting analysis was carried out by incubation of the membrane with one of the following primary antibodies: anti-cytochrome c, anti-GFP, anti-Actin and anti-caspase-8 (Santa Cruz Biotechnology); anti-caspase-3 (Cell Signaling); anti-Bcl-2 (Sigma-Aldrich), and followed by incubation with horseradish peroxidase conjugated secondary antibodies (Santa Cruz Biotechnology). Immune complexes were detected by the enhanced chemiluminescence system according to the manufacturer's instructions (ECL; GE Healthcare).

\section{Flow cytometric analysis}

To identify sub-G1 DNA region (below the G0/G1 peak) that is indicative of cells undergoing apoptosis, the drug-treated cells were harvested and fixed with $70 \%$ ethanol. The fixed cells were digested with RNase A, stained with propidium iodide and analyzed by flow cytometer (FACScan; BD Biosciences).

\section{Statistical analysis}

All the data in this study were expressed as mean \pm standard error (SE) from at least three independent experiments and the comparisons of means were performed using the paired $t$-test. A value of $p<0.05$ was considered statistically significant.

\section{Results}

\section{Cleavage of Bcl-2 protein during cisplatin-induced apop- tosis}

Cisplatin is a potent inducer of apoptosis and widely used as a chemotherapeutic agent [17]. It was used here to induce apoptosis in human hepatocarcinoma BEL-7404 cells (Figure 1A). We examined the changes of Bcl-2 protein in cisplatin-treated cells by Western-blotting analysis. Two cleaved bands of about $23 \mathrm{kDa}$ were clearly detected by the anti-human Bcl-2 monoclonal antibody when the cells were treated with $10 \mu \mathrm{g} / \mathrm{ml}$ or higher concentrations of cisplatin (Figure 1B). Moreover, the drug-induced cleavage of Bcl2 could be inhibited by preincubation with a pan-caspase inhibitor z-VAD-fmk (Figure 1B, last lane).

To confirm that the Bcl-2 protein was cleaved to produce two fragments of about $23 \mathrm{kDa}$ in the presence of cisplatin, we transfected $\mathrm{CHO}$ cells with EGFP-fused human Bcl-2 (hBcl-2). The expression of exogenous hBcl-2 was confirmed by Western-blotting with anti-hBcl-2 and anti-GFP, respectively (Figure 2A). There was no interference of endogenous Bcl-2 of CHO cells in Western-blotting analysis because of no cross-reaction between the anti-hBcl-2 
$\mathrm{mAb}$ and the hamster Bcl-2 protein. After treatment with cisplatin for $24 \mathrm{~h}$, two cleaved fragments of EGFP-fused human Bcl-2 protein were detected (Figure 2A, upper panel). When the drug treatment was prolonged to $48 \mathrm{~h}$, the intensity of cleaved fragments increased (Figure 2A, upper panel). Both cleaved products could be eliminated by preincubation with the inhibitor $\mathrm{z}-\mathrm{VAD}$-fmk (Figure $2 \mathrm{~A}$, upper panel), indicating that these two small fragments of Bcl-2 were likely generated via cleavage by caspases. Although the level of total Bcl-2 was higher in the cisplatin-treated cells compared to the untreated cells (Figure 2A, upper panel, compare lane 3, 4, 6, 7 with lane 2), there was no significant difference in Bcl-2 abundance between the 24 h-drug-treated cells and 48h-drug-treated cells, suggesting that the increment of Bcl-2 cleavage at $48 \mathrm{~h}$ is determined by the increased activity of caspases, but not

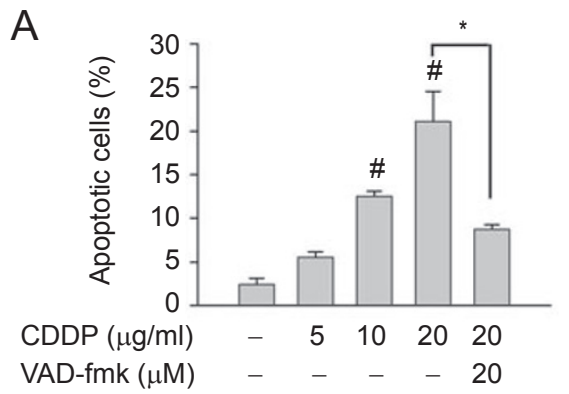

B

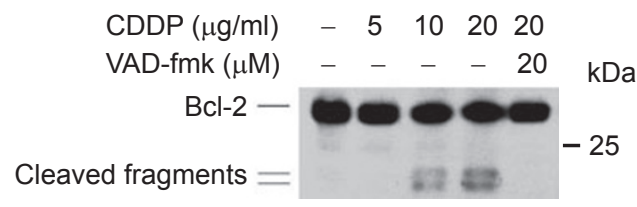

Figure 1 Bcl-2 cleavage in BEL-7404 cells. (A) Apoptosis induced by cisplatin (CDDP) in BEL-7404 cells. After treatment with cisplatin at different concentrations for $24 \mathrm{~h}$, the percentage of cells with a sub-G1 DNA content (taken as apoptotic cells) were measured by flow cytometry. The apoptotic cells were reduced by $1 \mathrm{~h}$ of preincubation with a pan-caspase inhibitor $\mathrm{z}-\mathrm{VAD}-\mathrm{fmk}$ (Promega). ${ }^{\#} p<0.05$, compared with control; ${ }^{*} p<0.05$, paired t-test. (B) Two fragments of Bcl-2 cleavage were detected in cisplatintreated BEL-7404 cells, and such cleavage was inhibited by z-VAD-fmk. The samples were separated by SDS-PAGE on an $12 \%$ acrylamide gel and detected with anti-hBcl-2 mAb.

A

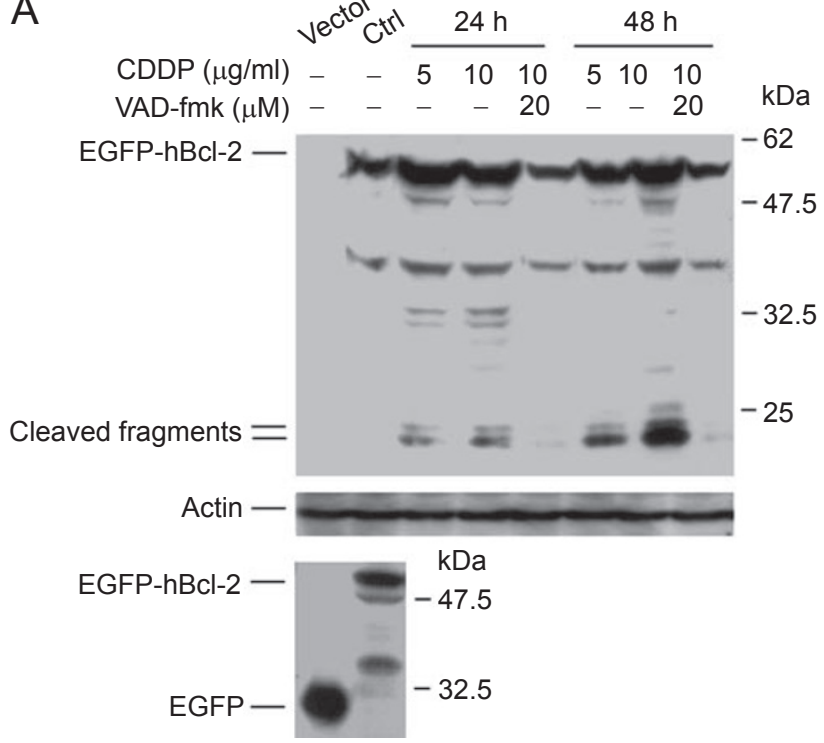

B

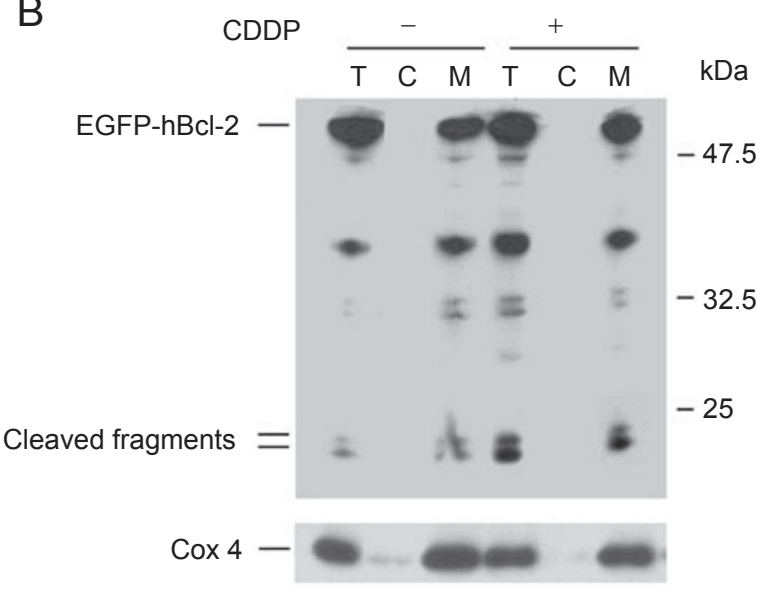

Figure 2 Bcl-2 cleavage in hBcl-2-overexpressing CHO cells. (A) The overexpression of EGFP-fused hBcl-2 protein was confirmed by Western-blotting analysis using anti-hBcl-2 and anti-GFP antibodies, respectively. Two fragments of Bcl-2 cleavage were detected in $\mathrm{hBcl}-2$ transfected $\mathrm{CHO}$ cells in the presence of cisplatin (CDDP), and such cleavage was inhibited by preincubation with z-VADfmk. Actin was used as sample-loading control. (B) The subcellular location of cleaved fragments. After drug treatment, cells were fractionated as described in Materials and Methods. Total cell lysates (T), cytosolic fractions (C) and mitochondrion-containing fractions (M) were subjected to Western-blotting. Cytochrome c oxidase IV (Cox 4) was shown as a quality control for fractionations. 
by the expression level of excessive Bcl-2.

Bcl-2 possesses a C-terminal transmembrane domain that localizes the protein to mitochondrial membrane [18]. If the cleavage is executed at $\mathrm{C}$-terminus, the truncated $\mathrm{Bcl}-2$ protein must be released from the membrane. To analyze the location of the truncated products in cells, we fractionated $\mathrm{Bcl}-2$ over-expressing $\mathrm{CHO}$ cells to obtain cytosolic and mitochondrion-containing fractions by the digitonin-permeabilization method (see Materials and Methods). The results showed that drug-induced cleaved fragments of Bcl-2 remained in the mitochondrion-fractions (Figure 2B), suggesting that they retained the $\mathrm{C}$-terminus of $\mathrm{Bcl}-2$ but lost the N-terminus.

\section{Determination of the cleavage sites in Bcl-2 protein}

There are several aspartic acid residues in the $\mathrm{N}$-terminus of $\mathrm{hBcl}-2$ protein, $\left(\mathrm{Asp}^{10}, \mathrm{Asp}^{31}, \mathrm{Asp}^{34}\right.$ and $\left.\mathrm{Asp}^{64}\right)$ that are candidate cleavage sites for caspases (Figure $3 \mathrm{~A}$ ). However, only the cleavages at $\mathrm{Asp}^{31}$ and $\mathrm{Asp}^{34}$ would make Bcl-2 protein lose its $\mathrm{BH} 4$ domain (Figure $3 \mathrm{~A}$ ) and yield two close fragments of about $23 \mathrm{kDa}$. It has been reported that $\mathrm{Asp}^{34}$ is the substrate site of caspase-3 [12]. We then tried to identify the actual cleavage sites in $\mathrm{hBcl}-2$ by constructing several mutants in which $\mathrm{Asp}^{31}$ (D31A) and $\mathrm{Asp}^{34}$ (D34A) were substituted by alanine, respectively or simultaneously (D31,34A). Constructs expressing the wild-type and mutant forms were transfected into $\mathrm{CHO}$ cells. After cisplatin treatment, the D34A mutant protein was cleaved to yield the larger fragment, whereas the D31A mutant protein was slightly cleaved to yield the smaller fragment (Figure 3B). However, cleavages were not detected in the D31,34A double mutant (Figure 3B). These results indicated that Bcl-2 protein was cleaved at both sites of $\mathrm{Asp}^{31}$ and $\mathrm{Asp}^{34}$ $\left(\mathrm{YEWD}^{31} \downarrow \mathrm{AGD}^{34} \downarrow \mathrm{V}\right)$ as expected.

Then $\mathrm{CHO}$ cells overexpressing wild-type or mutant Bcl-2 molecules were analyzed for the effects of these mutations on apoptosis under cisplatin treatment. Both D31A and D34A exhibited enhanced protective effects on apoptosis compared with wild-type Bcl-2 (Figure 3C). The decrease of apoptosis was more obvious in cells expressing the D31,34A double mutant in which the cleavage was completely abolished (Figure 3C). These results suggest that cleavages of $\mathrm{Bcl}-2$ promote cisplatin-induced apoptotic cell death.

Involvement of caspase- 8 and caspase-3 in the cisplatininduced Bcl-2 cleavage

Since the DAGD ${ }^{34}$ sequence fits with the caspase-3-

A BH4 domain
Human Bcl-2
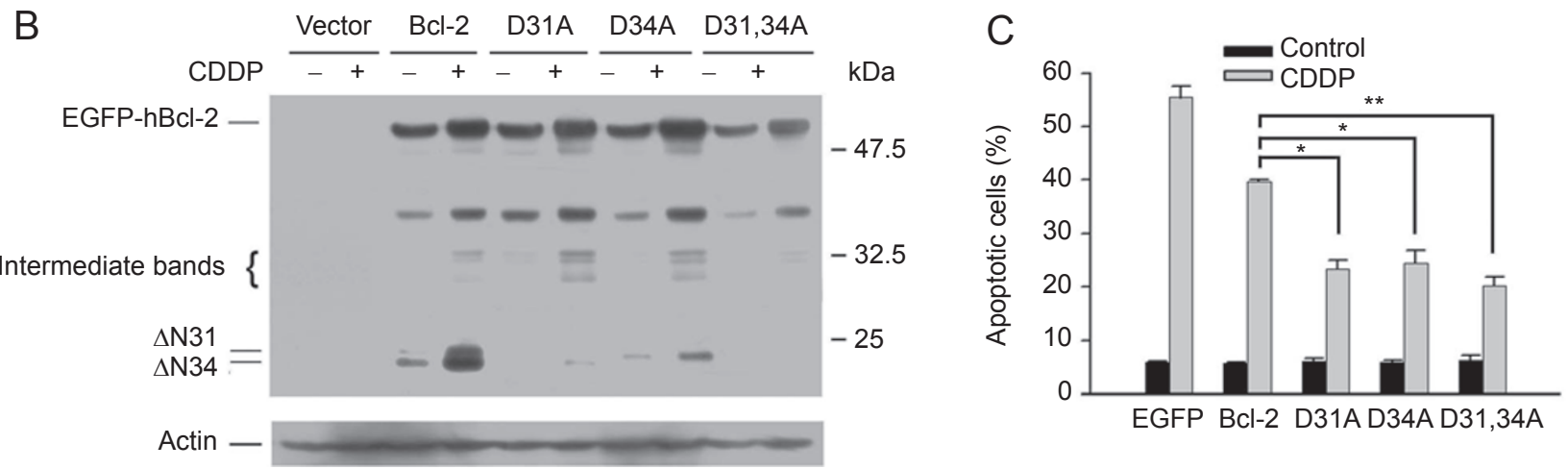

Figure 3 Identification of the cleavage sites. (A) Amino acid sequence of $\mathrm{NH}_{2}$-terminus of human Bcl-2 protein containing two putative caspase cleavage sites: $\mathrm{Asp}^{31}$ and $\mathrm{Asp}^{34}$. (B) The effect of site-directed mutations of Asp ${ }^{31}$ and Asp ${ }^{34}$ on Bcl-2 cleavage. CHO cells overexpressing wild-type Bcl-2 or mutant Bcl-2s (D31A, D34A and D31, 34A) were treated with $10 \mu \mathrm{g} / \mathrm{ml} \mathrm{cisplatin}$ (CDDP) for $24 \mathrm{~h}$ and then analyzed by Western-blotting assay. Actin was used as the sample-loading control. (C) The effect of cleavage-defective mutant Bcl-2s on cisplatin-induced apoptosis. Cells with sub-G1 DNA contents after 48 h CDDP treatment were measured by flow cytometry. ${ }^{*} p<0.05$ and ${ }^{* *} p<0.01$, paired t-test. 
A

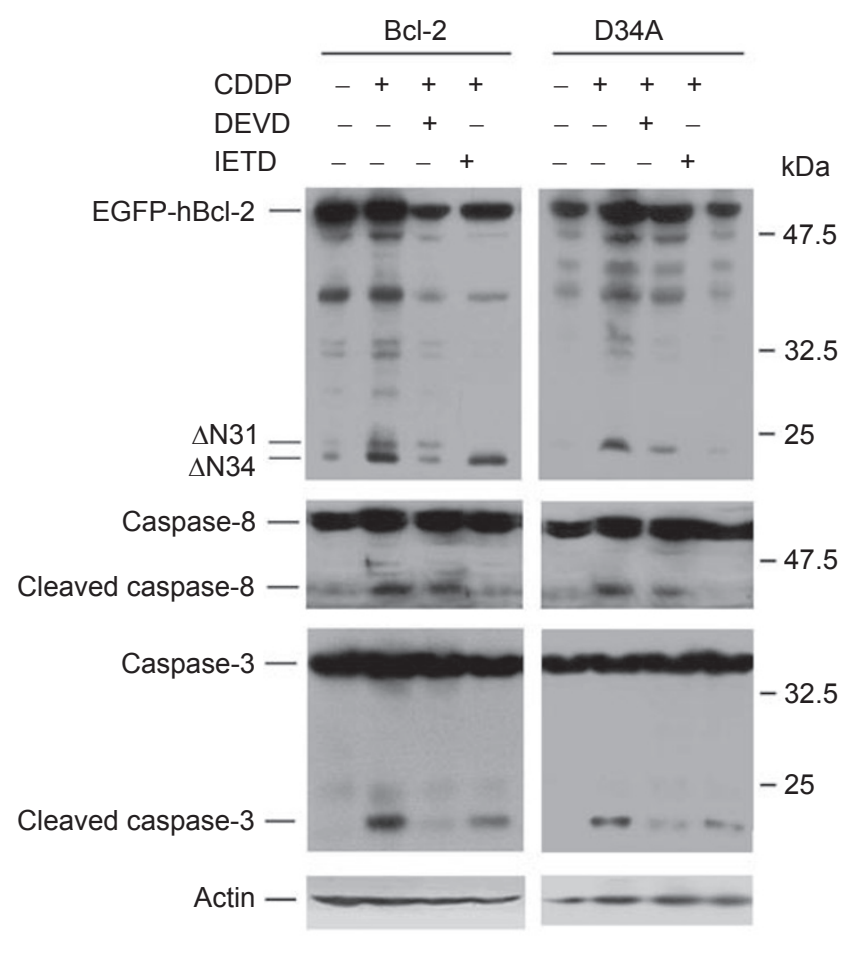

B
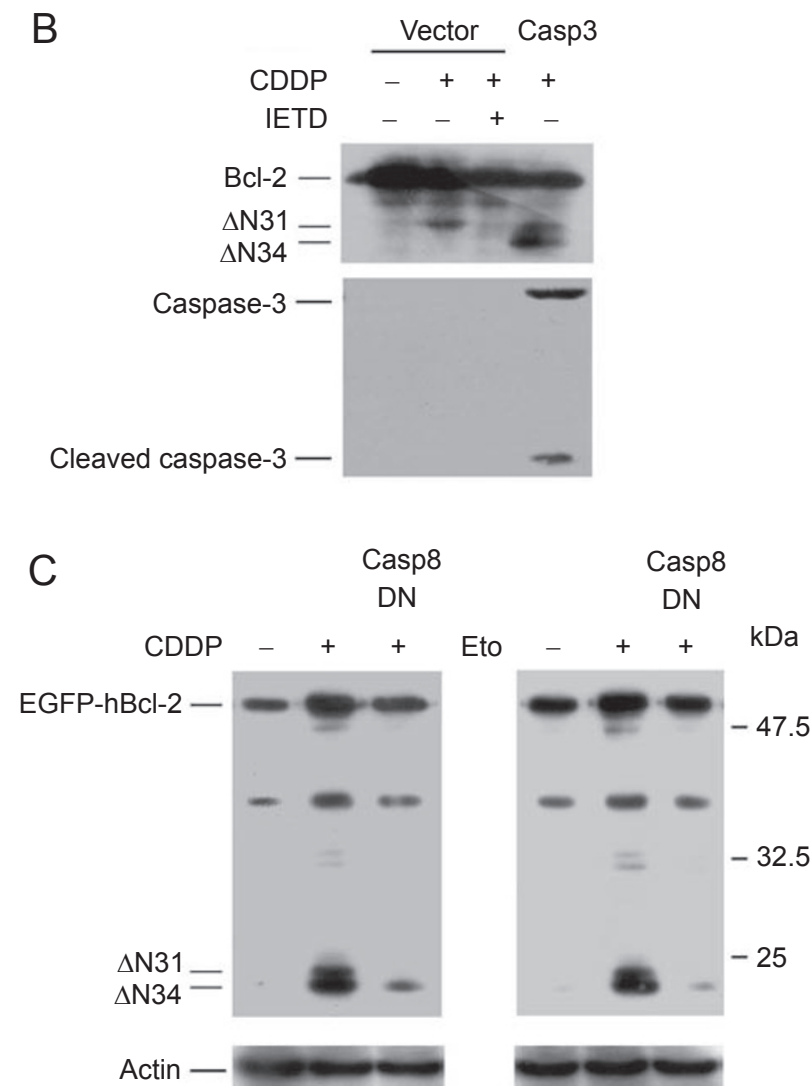

Figure 4 Involvement of caspase- 8 and caspase-3 in Bcl-2 cleavage. (A) The two cleavages of Bcl-2 were inhibited by the caspase8 inhibitor CP-IETD-cho (Calbiochem) and caspase-3 inhibitor CP-DEVD-cho (Calbiochem), respectively. CHO cells expressing wild-type Bcl-2 or D34A mutant were preincubated with $20 \mu \mathrm{M}$ inhibitors for $1 \mathrm{~h}$ and treated with $10 \mu \mathrm{g} / \mathrm{ml}$ cisplatin (CDDP) for 24 $\mathrm{h}$. The activities of caspase- 8 and caspase- 3 in the absence/presence of the inhibitors were examined by Western-blotting analysis. Actin was used as the sample-loading control. (B) The cleavage in caspase-3 reconstituted MCF-7 cells. MCF-7 cells transfected with the caspase-3 expression construct or control vector were treated with $10 \mu \mathrm{g} / \mathrm{ml}$ CDDP for $36 \mathrm{~h}$, or the caspase-3-deficient MCF-7 cells were preincubated with $20 \mu \mathrm{M}$ IETD-cho before the CDDP-treatment. (C) The cleavage in dominant-negative caspase- 8 (Casp8 DN) overexpressing cells. The stable transfectants and control cells were transiently transfected with wild-type Bcl-2, then treated with $10 \mu \mathrm{g} / \mathrm{ml} \mathrm{CDDP}$ for $24 \mathrm{~h}$ (left panel) or $100 \mu \mathrm{M}$ etoposide (Eto) for $48 \mathrm{~h}$ (right panel), respectively. Actin was used as the sample-loading control.

like protease recognition motif DXXD and mutagenesis analysis suggested that $\mathrm{Asp}^{31}$ is required for cleavage at $\mathrm{Asp}^{34}$ (Figure 3B), we hypothesized that cleavage at $\mathrm{Asp}^{34}$ is executed by a caspase-3-like protease. The sequence of YEWD $^{31}$ is not an optimal target site for caspase-3 [19], and it is likely to be cleaved by an unidentified caspase. The large fragment (named $\Delta \mathrm{N} 31$ ) was produced in early period $(24 \mathrm{~h})$, while an increasing amount of the small fragment (named $\Delta \mathrm{N} 34$ ) was detected upon longer drug-treatment $(48 \mathrm{~h})$ (Figure 2A), suggesting that cleavage at $\mathrm{Asp}^{31}$ was carried out by a caspase preceding caspase-3 activation. Our previous study reported caspase- 8 mediated cleavage of Bcl-2, which was independent of caspase-3 [16]. To explore if caspase- 8 and caspase- 3 were involved in cispla- tin-induced Bcl-2 cleavage, we used the caspase- 8 specific inhibitor IETD-cho and the caspase-3 specific inhibitor DEVD-cho to suppress the corresponding proteases activities $[20,21]$. As shown in Figure 4A, $\Delta$ N34 was reduced to background level in the presence of DEVD-cho (lane 3), and $\Delta \mathrm{N} 31$ was eliminated when cells overexpressing either wild-type Bcl-2 (lane 4) or mutant D34A (lane 8, in which $\triangle \mathrm{N} 34$ is absent) were pre-incubated with IETD-cho.

MCF-7 is a cell line deficient of caspase-3 [22], which was used to further investigate the mechanism of Bcl-2 cleavage. We reconstituted caspase-3 in MCF-7 cells to restore its function. When cells transfected with vector control were treated with cisplatin, only one cleaved band (should be $\Delta \mathrm{N} 31$ ) was detected, and its production could be 
inhibited by IETD-cho. In caspase-3 reconstituted MCF-7 cells, besides the former cleaved band, a smaller cleaved band (should be $\Delta \mathrm{N} 34$ ) was produced, consistent with the notion that it was generated by caspase-3 (Figure 4B).

To further confirm the role of caspase- 8 in Bcl-2 cleavage, a $\mathrm{CHO}$ cell line stably expressing dominant-negative (DN) caspas-8 (16) was used, in which caspase-8 activity was specifically inhibited. After cisplatin treatment, the cleaved product $\Delta \mathrm{N} 31$ was not detected, while $\Delta \mathrm{N} 34$ was partially reduced (Figure $4 \mathrm{C}$, left panel). To find out if such caspase-8-mediated cleavage was specific to cisplatin induced apoptosis, we further used another chemotherapeutic reagent etoposide as the apoptosis inducer, which activates the caspase-8-mediated apoptotic pathway [23, 24]. Similar results were obtained (Figure 4C, right panel), suggesting that this kind of caspase-8-mediated cleavage of Bcl-2 occurs generally once caspase- 8 is activated by apoptotic stresses.
Taken together, these results argue that caspase- 8 is involved in the cleavage at $\mathrm{Asp}^{31}$, while cleavage at $\mathrm{Asp}^{34}$ might be mediated by caspase-3.

\section{Acceleration of apoptosis by the cleaved Bcl-2 fragments}

Previous work reported that the single cleaved fragment of $\mathrm{Bcl}-2$ promoted apoptosis $[12,16]$. To explore the function of these two cleaved fragments of Bcl-2, we constructed vectors individually expressing each of the cleaved $\mathrm{Bcl}-2$ fragments: $\Delta \mathrm{N} 31$ (losing amino acids 1-31), $\Delta \mathrm{N} 31 \mathrm{D} 34 \mathrm{~A}$ $(\Delta \mathrm{N} 31$ with a D34A mutation), and $\Delta \mathrm{N} 34$ (losing amino acids 1-34). These constructs were transiently transfected into $\mathrm{CHO}$ cells. Western-blotting analysis showed that the positions of truncated proteins on gel were consistent with the size of the products cleaved from EGFP-Bcl-2 (Figure $5 \mathrm{~A})$. In response to cisplatin stimulation, the expression of truncated $\mathrm{Bcl}-2$ proteins were upregulated but no further cleavage at $\mathrm{Asp}^{34}$ was detected in $\Delta \mathrm{N} 31$ (Figure 5A). Fur-

A
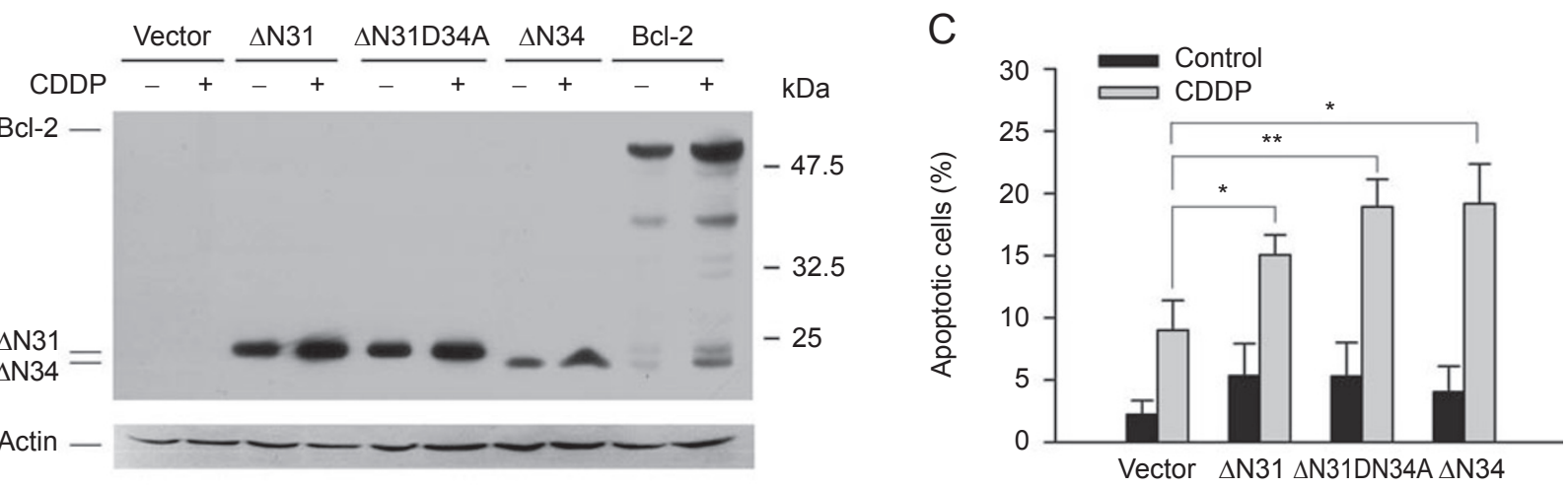

B

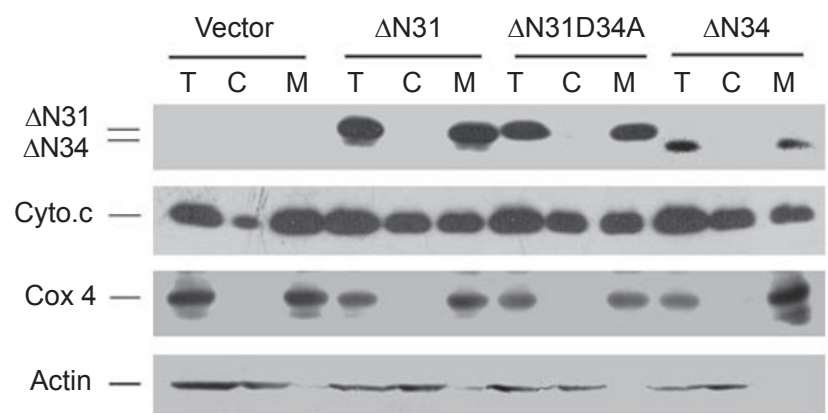

Figure 5 The truncated Bcl-2 proteins promoted cytochrome c release and accelerated apoptosis. (A) Expression of truncated Bcl-2 proteins including $\Delta \mathrm{N} 31$ (amino acids $32-239), \Delta \mathrm{N} 31 \mathrm{D} 34 \mathrm{~A}(\Delta \mathrm{N} 31$ with a D34A mutation) and $\Delta \mathrm{N} 34$ (amino acids $35-239)$. Actin was used as the sample-loading control. (B) The truncated Bcl-2 fragments promoted cytochrome $\mathrm{c}$ release. After treatment with 10 $\mu \mathrm{g} / \mathrm{ml}$ cisplatin (CDDP) for $12 \mathrm{~h}$, the apoptotic cells were harvested and fractionated. Total cell lysates (T), cytosolic fractions (C) and mitochondrion-containing fractions $(\mathrm{M})$ were subjected to Western-blotting. Cytochrome c oxidase IV (Cox 4) and actin were shown as mitochondrial and cytosolic markers to control the quality of fractionation. (C) The truncated Bcl-2 proteins accelerated cell death. Cells with sub-G1 DNA contents were analyzed by flow cytometry. ${ }^{*} p<0.05$ and ${ }^{* *} p<0.01$, paired t-test. 
thermore, cell fractionation assay showed that the expressed truncated proteins were all detected in the mitochondrionfractions (Figure 5B), suggesting that they could function like naturally cleaved Bcl-2 fragments.

We then examined the apoptotic status of cisplatintreated $\mathrm{CHO}$ cells overexpressing these truncated Bcl-2 proteins. The percentage of apoptotic cells was significantly increased by transfection with any of the N-terminal truncated Bcl-2, comparing to the vector control (Figure 5C). Consistent with this, cell fractionation assay showed that more cytochrome $\mathrm{c}$ was released from mitochondria to cytosol in cells overexpressing the truncated proteins (Figure 5B). These results indicated that both forms of the cleaved Bcl-2 fragments, $\Delta \mathrm{N} 31$ and $\Delta \mathrm{N} 34$, increased the sensitivity of cells to apoptotic stimuli.

\section{Discussion}

It was previously reported that Bcl-2 could be cleaved under apoptotic stimuli, which can inactivate its anti-apoptotic activity or even convert Bcl-2 to a Bax-like effector $[11,12]$. It was proposed that Bcl-2 cleavage could act as a positive feedback amplification loop that accelerates the apoptotic process $[25,26]$. In the course of examining the transformation of Bcl-2 protein, we found two cleaved fragments around $23 \mathrm{kDa}$ that were recognised by an anti-human $\mathrm{Bcl}-2 \mathrm{mAb}$ against an epitope within amino acids 41-54 (Figure 3A). It was reported that $\mathrm{Asp}^{34}$ was the cleavage site in Bcl-2 for caspase- 3 and $\mathrm{Asp}^{31}$ was also required for this cleavage [12], which was supported by our point-mutation experiments (Figure 3B). It was consistent with the fact that caspase-3 preferentially cleaves substrates containing aspartic acid residues at both $\mathrm{P} 1$ and P4 positions (DXXD $\downarrow$ ).

Our present studies also showed that a larger fragment $(\Delta \mathrm{N} 31)$ was cleaved from the Bcl-2-D34A mutant in the presence of cisplatin (Figure 3B, panel D34A), suggesting other caspases that do not need the cleavage site of $\mathrm{Asp}^{34}$ were also involved in the drug-induced cleavage of Bcl-2. Besides caspase- 3 , other caspases such as caspase-2, -6, $-7,-8,-9$ and -10 also participate in the apoptotic process [19]. Since we have previously shown a caspase- 8 mediated cleavage of Bcl-2, which was independent of caspase-3 [16], we proposed that $\mathrm{Asp}^{31}$ may be the potential caspase- 8 cleavage site. In support of this, the generation of $\Delta \mathrm{N} 31$ was eliminated when caspase- 8 activity was inhibited either by the caspase- 8 specific inhibitor (Figure 4A) or by the dominant-negative caspase-8 (Figure 4C). Further experiments also supported this conclusion since the larger cleaved fragment of Bcl-2 was produced in MCF-7 cells that lack caspase-3 activity [14], and its production was inhibited by the caspase- 8 specific inhibitor (Figure 4B).
The coactions by two different caspases may accelerate the process of Bcl-2 cleavage and rapidly amplify apoptotic signals.

There are several protein bands around $32.5 \mathrm{kDa}$ detected by Western-blotting in cisplatin-treated cells (Figure 2A, $3 \mathrm{~B}$ and $4 \mathrm{~A}$ ), among which some were faintly visible in cells expressing the D31,34A double mutant of Bcl-2 (Figure $3 \mathrm{~B}$ ) or in cells treated with caspases inhibitors (Figure 4A). They might be produced by cleavages at certain aspartic acid residues within EGFP that satisfy the recognition sites of certain caspases. Accompanied with the reduction of apoptosis, the activation of caspases would also be inhibited by overexpressing the D31,34A mutant, which would result in weakening of these intermediate bands. Another possible explanation is that these bands are due to nonspecific recognition by the $\mathrm{Bcl}-2$ antibody used in these experiments since these bands were also seen in some cells without cisplatin treatment (Figure 2B, lane1 and 3; Figure 4A, lane 1).

The Bcl-2 protein loses its fourth region of homology domain (BH4) when cleaved at the loop domain. The BH4 domain, conserved among anti-apoptotic Bcl-2 family members, is required for homodimerization or heterodimerization with other apoptotic effectors $[13,27]$. We transfected different truncated $\mathrm{Bcl}-2$ constructs $(\Delta \mathrm{N} 31$, $\triangle \mathrm{N} 31 \mathrm{D} 34 \mathrm{~A}, \Delta \mathrm{N} 34)$ into $\mathrm{CHO}$ cells. All of them promoted the process of drug-induced apoptosis (Figure 5C), indicating that $\mathrm{BH} 4$ domain is crucial for the protective activity of $\mathrm{Bcl}-2$. Removing BH4 domain may lead to the exposure of the pro-apoptotic $\mathrm{BH} 3$ domain of $\mathrm{Bcl}-2$, reminiscent of the cleavage on Bid [28]. The truncated Bcl-2 facilitates the release of cytochrome c (Figure 5B). The cytosolic cytochrome $\mathrm{c}$ together with Apaf-1 activates caspase-9, which in turn activates caspase-3 [29]. Thus the caspase cascade could be amplified by the cleavage of Bcl-2.

\section{Acknowledgment}

This work was supported by grants of National Natural Science Foundation of China (\#30230110, \#0637S12442, \#30670433) to Jiarui Wu.

\section{References}

1 Jacobson MD, Weil M, Raff MC. Programmed cell death in animal development. Cell 1997; 88:347-354.

2 Wyllie AH. Apoptosis: an overview. Br Med Bull 1997; 53:451465.

3 Zimmermann KC, Bonzon C, Green DR. The machinery of programmed cell death. Pharmacol Ther 2001; 92:57-70.

4 Green DR. At the gates of death. Cancer Cell 2006; 9:328-330.

5 Yang J, Liu X, Bhalla K, et al. Prevention of apoptosis by Bcl2: release of cytochrome $c$ from mitochondria blocked. Science 1997; 275:1129-1132. 
6 Slee EA, Adrain C, Martin SJ. Serial killers: ordering caspase activation events in apoptosis. Cell Death Differ 1999; 6:10671074.

7 Fischer U, Janicke RU, Schulze-Osthoff K. Many cuts to ruin: a comprehensive update of caspase substrates. Cell Death Differ 2003; 10:76-100.

8 Li H, Zhu H, Xu CJ, Yuan J. Cleavage of BID by caspase 8 mediates the mitochondrial damage in the Fas pathway of apoptosis. Cell 1998; 94:491-501.

9 Tewari M, Quan LT, O'Rourke K, et al. Yama/CPP32 beta, a mammalian homolog of CED-3, is a CrmA-inhibitable protease that cleaves the death substrate poly(ADP-ribose) polymerase. Cell 1995; 81:801-809.

10 Wolf BB, Schuler M, Echeverri F, Green DR. Caspase-3 is the primary activator of apoptotic DNA fragmentation via DNA fragmentation factor-45/inhibitor of caspase-activated DNase inactivation. J Biol Chem 1999; 274:30651-30656.

11 Grandgirard D, Studer E, Monney L, et al. Alphaviruses induce apoptosis in Bcl-2-overexpressing cells: evidence for a caspasemediated, proteolytic inactivation of Bcl-2. EMBO J 1998; 17:1268-1278.

12 Cheng EH, Kirsch DG, Clem RJ, et al. Conversion of Bcl-2 to a Bax-like death effector by caspases. Science 1997; 278:19661968.

13 Hirotani M, Zhang Y, Fujita N, Naito M, Tsuruo T. NH2-terminal $\mathrm{BH} 4$ domain of $\mathrm{Bcl}-2$ is functional for heterodimerization with Bax and inhibition of apoptosis. J Biol Chem 1999; 274:2041520420.

14 Kim YM, Kim TH, Seol DW, Talanian RV, Billiar TR. Nitric oxide suppression of apoptosis occurs in association with an inhibition of Bcl-2 cleavage and cytochrome $c$ release. J Biol Chem 1998; 273:31437-31441.

15 Zhang XM, Lin H, Chen C, Chen BD. Inhibition of ubiquitinproteasome pathway activates a caspase-3-like protease and induces Bcl-2 cleavage in human M-07e leukaemic cells. Biochem J 1999; 340:127-133.

16 Zhu JB, Xiong L, Yu B, Wu JR. Apoptosis Induced by a New Member of Saponin Family Is Mediated through Caspase-8Dependent Cleavage of Bcl-2. Mol Pharmacol 2005; 68:18311838.

17 Sedletska Y, Giraud-Panis MJ, Malinge JM. Cisplatin is a DNA-damaging antitumour compound triggering multifactorial biochemical responses in cancer cells: importance of apoptotic pathways. Curr Med Chem Anticancer Agents 2005; 5:251265 .
18 Nguyen M, Millar DG, Yong VW, Korsmeyer SJ, Shore GC Targeting of Bcl-2 to the mitochondrial outer membrane by a $\mathrm{COOH}$-terminal signal anchor sequence. J Biol Chem 1993; 268:25265-25268.

19 Pratesi G, Perego P, Zunino F. Role of Bcl-2 and its post-transcriptional modification in response to antitumor therapy. Biochem Pharmacol 2001; 61:381-386.

20 Martin S, Phillips DC, Szekely-Szucs K, et al. Cyclooxygenase-2 inhibition sensitizes human colon carcinoma cells to TRAILinduced apoptosis through clustering of DR5 and concentrating death-inducing signaling complex components into ceramideenriched caveolae. Cancer Res 2005; 65:11447-11458.

21 Kwon KB, Kim EK, Han MJ, et al. Induction of Apoptosis by Radix Paeoniae Alba Extract through Cytochrome c Release and the Activations of Caspase-9 and Caspase-3 in HL-60 Cells. Biol Pharm Bull 2006; 29:1082-1086.

22 Janicke RU, Sprengart ML, Wati MR, Porter AG. Caspase-3 is required for DNA fragmentation and morphological changes associated with apoptosis. J Biol Chem 1998; 273:9357-9360.

23 Chandra D, Choy G, Deng X, et al. Association of active caspase 8 with the mitochondrial membrane during apoptosis: potential roles in cleaving BAP31 and caspase 3 and mediating mitochondrion-endoplasmic reticulum cross talk in etoposide-induced cell death. Mol Cell Biol 2004; 15:6592-6607.

24 Sohn D, Schulze-Osthoff K, Janicke RU. Caspase-8 can be activated by interchain proteolysis without receptor-triggered dimerization during drug-induced apoptosis. J Biol Chem 2005; 7:5267-5273.

25 Fadeel B, Zhivotovsky B, Orrenius S. All along the watchtower: on the regulation of apoptosis regulators. FASEB J 1999; 13:1647-1657.

26 Kirsch DG, Doseff A, Chau BN, et al. Caspase-3-dependent cleavage of Bcl-2 promotes release of cytochrome c. J Biol Chem 1999; 274:21155-21161

27 Reed JC, Zha H, Aime-Sempe C, Takayama S, Wang HG. Structure-function analysis of Bcl-2 family proteins. Regulators of programmed cell death. Adv Exp Med Biol 1996; 406:99-112.

28 McDonnell JM, Fushman D, Milliman CL, Korsmeyer SJ, Cowburn D. Solution structure of the proapoptotic molecule BID: a structural basis for apoptotic agonists and antagonists. Cell 1999; 96:625-634.

29 Li P, Nijhawan D, Budihardjo I, Srinivasula SM, Ahmad M, et al. Cytochrome $\mathrm{c}$ and dATP-dependent formation of Apaf-1/caspase9 complex initiates an apoptotic protease cascade. Cell 1997; 91:479-489. 